\title{
Etude physiologique de Kluyveromyces fragilis conséquence pour la production de levure sur lactosérum
}

\author{
par \\ G. MOULIN*, B. MALIGE** et P. GALZY*
}

Le lactosérum est un produit dont la composition varie suivant la provenance. Dans le cas des lactosérums doux, leur richesse en lactose a entraîné la mise au point de divers procédés de fabrication de levure aliment. De nombreux auteurs $[10,2,1,4,6]$ ont montré que les souches appartenant aux espèces Kluyveromyces fragilis et Kluyveromyces lactis sont les mieux adaptées à cette production. Dans le présent travail nous avons étudié les métabolismes respiratoire et fermentaire d'une souche de Kluyveromyces fragilis et les conséquences industrielles des mécanismes de régulation de son métabolisme.

\section{MATERIELS ET METHODES}

La souche utilisée est un Kluyveromyces fragilis.

Toutes les cultures sont faites en milieu liquide et agitées ( 80 oscillations par min ; amplitude $8 \mathrm{~cm}$ ), en erlenmeyers remplis au dixième de leur volume. La température est de $28^{\circ} \mathrm{C}$.

Le milieu utilisé est du yeast nitrogen base Difco ( 0,67 p. 100), additionné d'un substrat carboné.

L'étude des paramètres métaboliques a été réalisée suivant la méthode directe de Warburg [9]. Pour cela, des prélèvements sont effectués aux divers stades de la croissance. Après centrifugation et lavage au moyen de phosphate monopotassique $\mathrm{M} / 15, \mathrm{pH} 4,5$, les échanges respiratoire et fermentaire sont mesurés. Divers coefficients ont été déterminés :

* Chaire de Génétique et Microbiologie, E.N.S.A., place Viala - 34060 Montpellier cedex.

** Centre de Recherches des Fromageries Bel - 41100 Vendôme. 
- Qoz - vitesse de respiration d'un substrat, exprimée en microlitres d'oxygène consommé par heure et par milligramme de matière sèche de levure.

- $\mathrm{Q}_{00}^{\mathrm{N}}$ - vitesse de fermentation d'un substrat en anaérobiose stricte exprimée en microlitres de $\mathrm{CO}_{2}$ dégagé par heure et par milligramme de matière sèche.

- Q Q $\mathrm{Q}_{\mathrm{i}}^{\mathrm{a}}$ ferm. - vitesse de fermentation d'un substrat en présence d'air exprimée en microlitres de $\mathrm{CO}_{2}$ dégagé par heure et par milligramme de matière sèche.

Les vitesses de croissance ont été calculées à partir du temps de génération, déterminé graphiquement sur les courbes de croissance. Les courbes de croissance sont établies par la mesure de la variation de la densité optique au cours du temps.

Les rendements de croissance ont été étudiés en faisant varier la teneur en substrat carboné de 0,1 p. 100 à 2 p. 100. Les rendements sont déterminés en calculant la pente des droites de régression dans la zone où il y a proportionnalité entre la matière sèche produite et la quantité de substrat carboné mise au départ.

\section{RESULTATS EXPERIMENTAUX}

\section{Etude des paramètres métaboliques}

La multiplication de Kluyveromyces fragilis est réalisée en présence de yeast nitrogen base additionné d'un des substrats carbonés suivants : glucose, lactose, éthanol, acide lactique. Pour chacun de ces quatre types de culture, la variation des paramètres métaboliques est mesurée à l'appareil de Warburg, selon la technique décrite précédemment [7]. Nous avons testé les vitesse de respiration : $\mathrm{Q}_{\circ 2}$ glucose, $\mathrm{Q}$ lactose, $\mathrm{Q}$ galactose, $\mathrm{Q} \approx$ éthanol, $\mathrm{Q}$ L-lactique, $Q_{02}$ acide D-lactique. Nous avons également mesuré

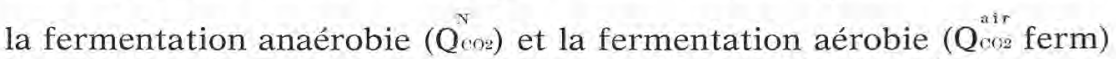
du glucose, du lactose et du galactose. L'ensemble des résultats est présenté sur les figures 1 à 4 .

Il n'y a pas d'effet glucose, c'est-à-dire une inhibition du métabolisme respiratoire $\left(Q_{02}\right)$ par le glucose [8]. L'activité respiratoire est, pour tous les substrats, adaptative : le $\mathrm{Q}_{\circ}$ est toujours plus élevé pour un substrat donné après culture sur ce même substrat.

De même le pouvoir fermentaire $\left(Q^{N}{ }^{N}\right)$ varie en fonction du substrat de croissance : le $\mathrm{Q}_{\mathrm{cor}}^{\mathrm{N}}$ glucose est plus élevé après culture sur glucose qu'après culture sur lactose ; l'inverse est observé pour le $\mathrm{Q}_{\mathrm{N}}^{\mathrm{N}}$ lactose. 

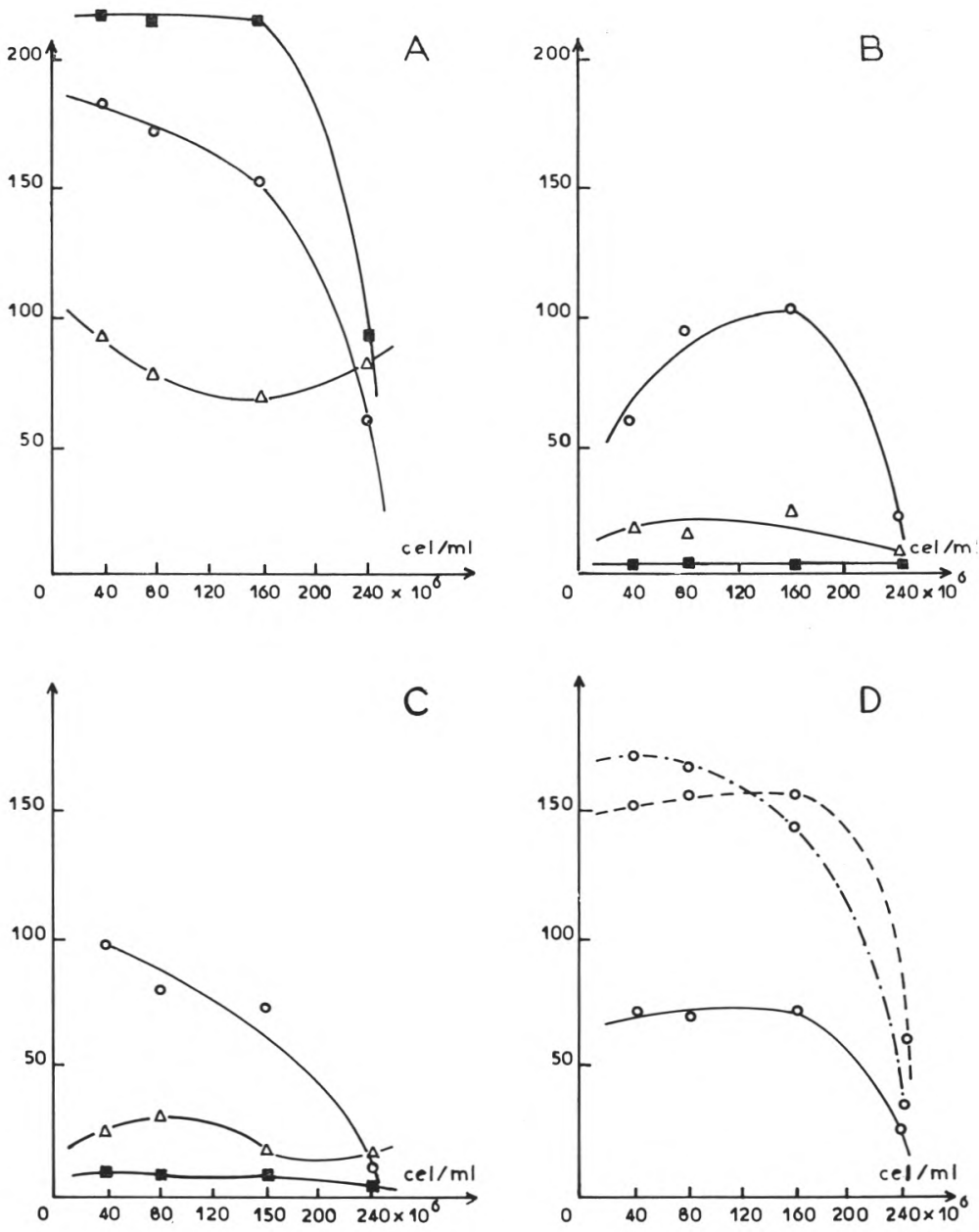

fig. 1

Etude des paramètres métaboliques de Kluyveromyces fragilis après culture sur glucose. Les mesures sont effectuées en présence de glucose (A), lactose (B), galactose (C), ( - ) éthanol, ( - $)$ acide L-lactique, (,-$)$ acide D-lactique (D).

Abscisse : Population cellulaire dans la culture en millions de cellules/ml.

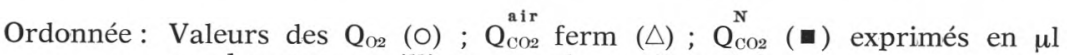
par heure par milligramme de matière sèche. 

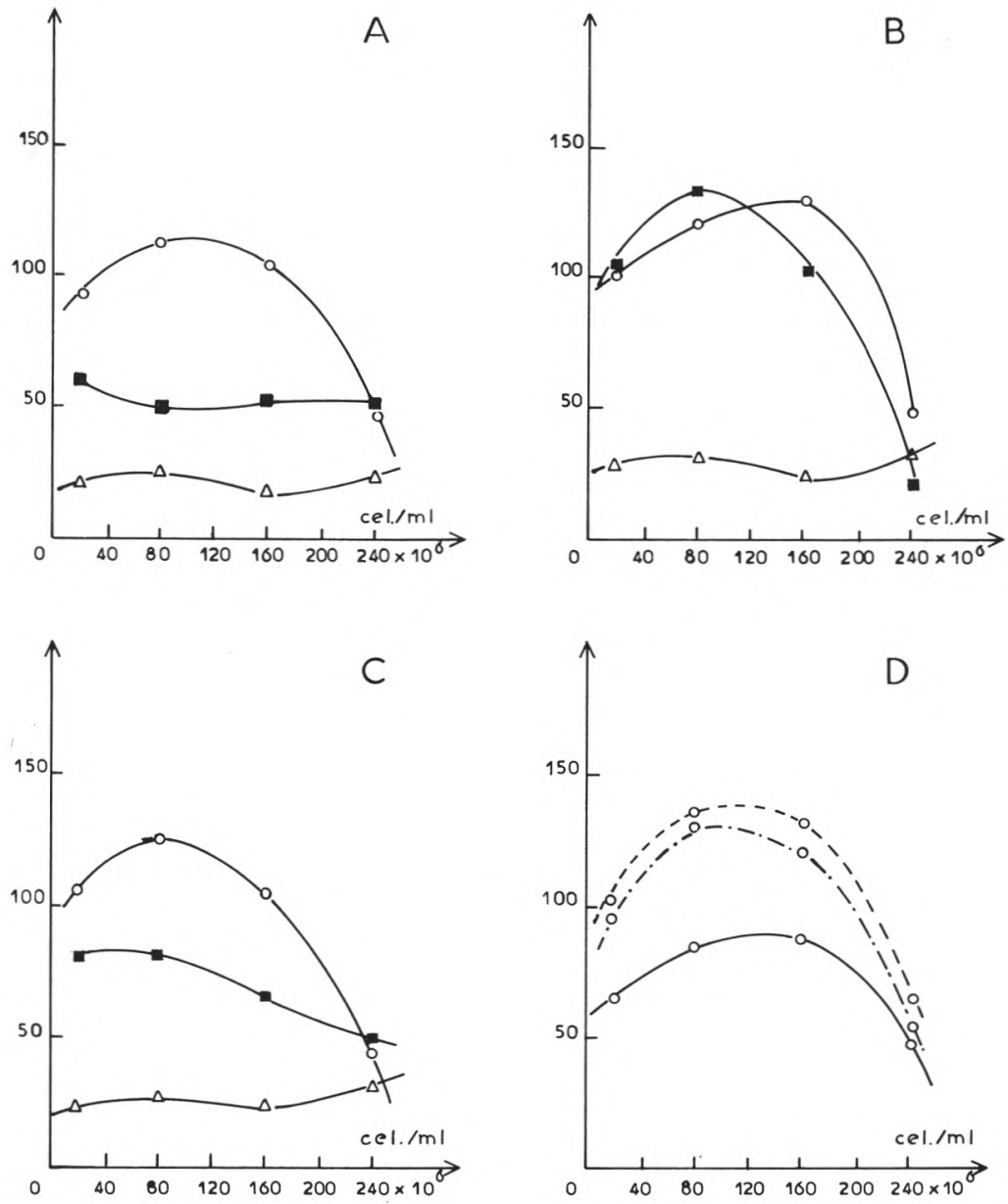

fig. 2

Etude des paramètres métaboliques de Kluyveromyces fragilis, après culture sur lactose. Les mesures sont effectuées en présence de glucose (A), lactose (B), galactose (C), ( $\longrightarrow$ ) éthanol, (- $)$ acide L-lactique, (-. $)$ acide D-lactique (D).

Abscisse : Population cellulaire dans la culture en millions de cellules/ml.

Ordonnée: Valeurs des $\mathrm{Q}_{\mathrm{O} 2}(\mathrm{O}) ; \mathrm{Q}_{\mathrm{CO} 2}^{\mathrm{air}}$ ferm $(\triangle) ; \mathrm{Q}_{\mathrm{CO} 2}^{\mathrm{N}}(\boldsymbol{\square})$ exprimés en $\mu \mathrm{l}$ par heure par milligramme de matière sèche. 

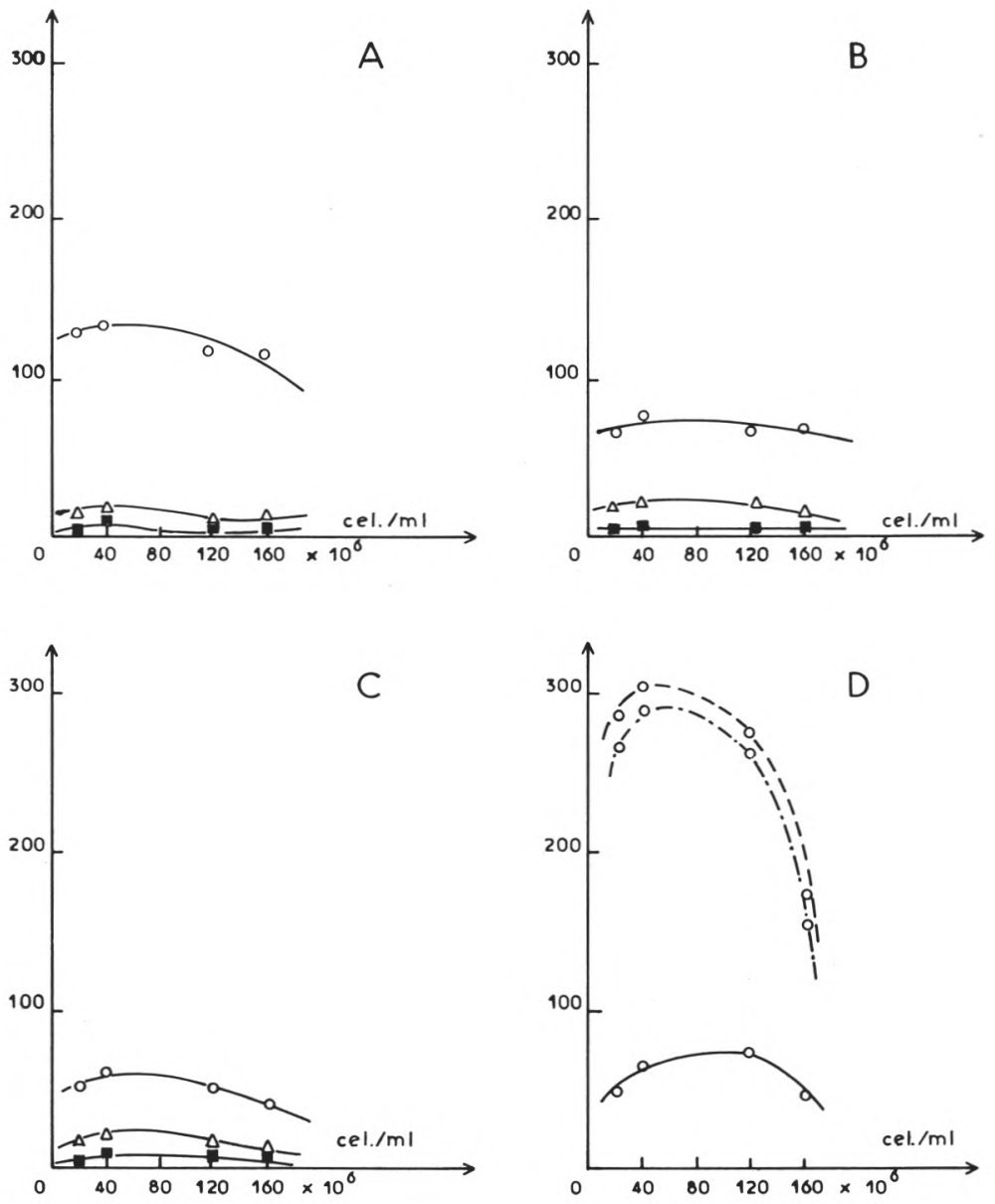

fig. 3

Etude des paramètres métaboliques de Kluyveromyces fragilis, après culture sur éthanol. Les mesures sont effectuées en présence de glucose $(A)$, lactose (B), galactose (C), ( - ) éthanol, ( - $)$ acide L-lactique, (-. -) acide D-lactique (D).

Abscisse : Population cellulaire dans la culture en millions de cellules/ml.

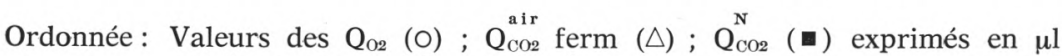
par heure par milligramme de matière sèche. 

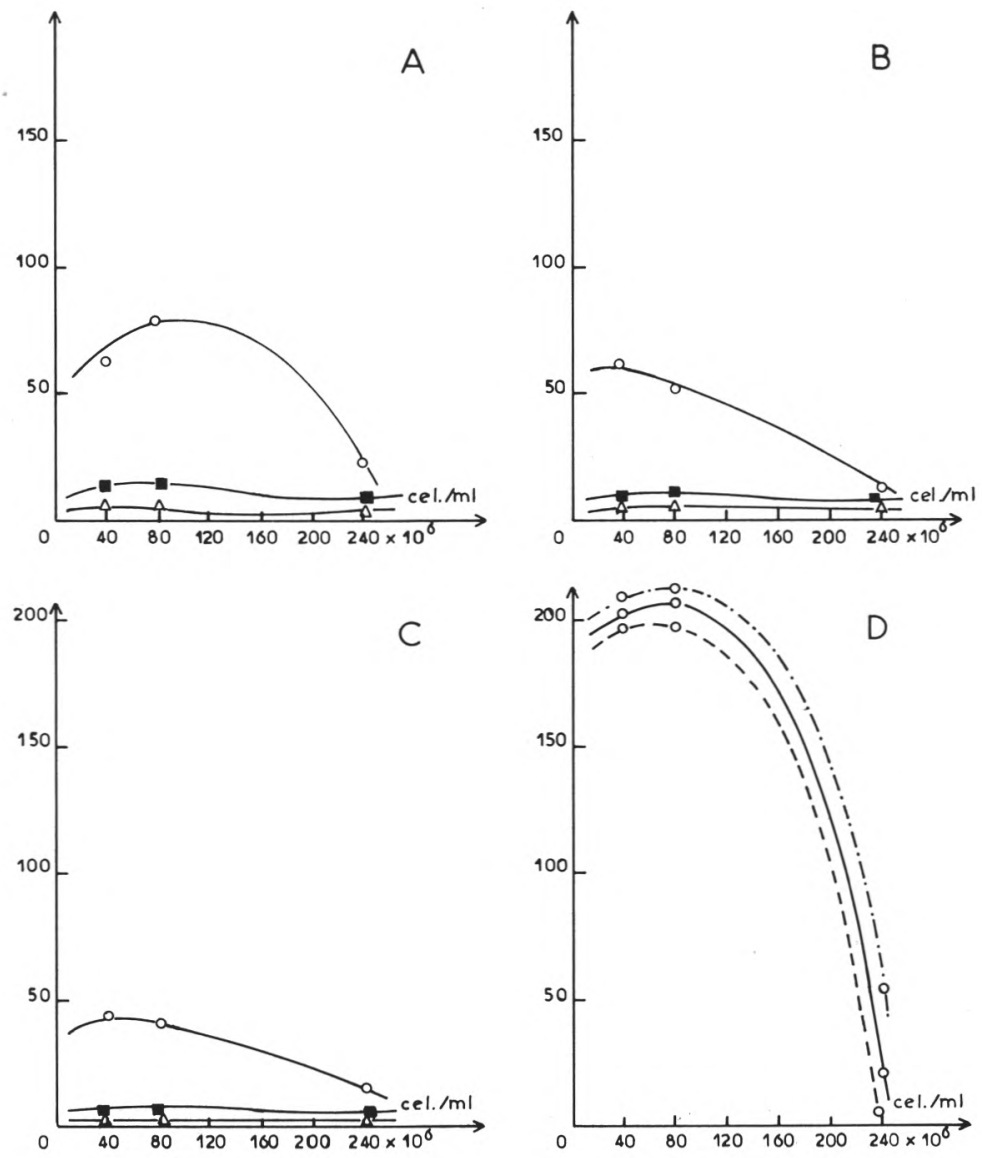

fig. 4

Etude des paramètres métaboliques de Kluyveromyces fragilis, après culture sur acide lactique. Les mesures sont effectuées en présence de glucose (A), lactose (B), galactose (C), ( - ) éthanol, $(--)$ acide L-lactique, (,-$)$ acide D-lactique (D).

Abscisse : Population cellulaire dans la culture en millions de cellules $/ \mathrm{ml}$.

Ordonnée: Valeurs des $\mathrm{Q}_{\mathrm{O} 2}(\mathrm{O}) ; \mathrm{Q}_{\mathrm{CO} 2}^{\mathrm{air}}$ ferm $(\Delta) ; \mathrm{Q}_{\mathrm{CO} 2}^{\mathrm{N}}(\boldsymbol{\bullet})$ exprimés en $\mu \mathrm{l}$ par heure par milligramme de matière sèche. 
Le métabolisme fermentaire $\left(\mathrm{Q}_{\mathrm{CO} 2}^{\mathrm{N}}\right)$ est toujours très faible après culture sur des substrats non fermentescibles (éthanol et acide lactique). Il est curieux de constater que le métabolisme fermentaire est, chez Kluyveromyces fragilis, de nature adaptative. Cette situation a déjà été décrite chez Kluyveromyces lactis [7]. La situation est nettement différente de celle observée chez les Saccharomyces.

La fermentation aérobie $\mathrm{Q}_{\mathrm{co} 2}^{\mathrm{a} \text { ir }}$ ferm du glucose, du lactose ou du galactose après culture sur chacun de ces substrats n'est jamais nulle et représente 20 à 40 p. 100 du niveau maximum de fermentation mesuré en anaérobiose stricte $\mathrm{Q}_{\mathrm{N}}^{\mathrm{N}}$. Bien que Kluyveromyces fragilis ne présente pas d'effet glucose la fermentation en présence d'air n'est pas nulle. L'effet Pasteur, inhibition du métabolisme fermentaire par l'oxygène de l'air, est ici très efficace et permet d'avoir un $\mathrm{Q}_{\mathrm{cos}}^{\mathrm{a} r}$ fermentaire très inférieur au $\mathrm{Q}^{\mathrm{N}} \mathrm{cos}$. Cependant, la fermentation n'étant jamais nulle, en présence de lactose, une accumulation d'éthanol est difficilement évitable.

\section{Etude des vitesses et rendement de croissance}

Cinq substrats potentiels ont été testés : glucose, lactose, galactose, acide DL lactique et éthanol. Les temps de génération, les taux népériens de la croissance ainsi que les rendements de la transformation du substrat en matière sèche sont fournis dans le tableau 1. Les résultats obtenus montrent que la souche se multiplie bien sur glucose, lactose et galactose. Par contre dans le cas de l'acide lactique et surtout dans le cas de l'éthanol les temps de génération sont plus élevés. Cette première observation montre que dans le cas de sérum acide contenant du lactose et de l'acide lactique, la transformation complète de ces deux substrats exigera de diminuer notablement le taux de dilution du fermenteur. Par contre, le galactose pourra être utilisé à des vitesses analogues au lactose ; sa présence est possible après certaines fabrications fromagères. L'éthanol est également utilisé lentement ce qui est gênant, compte tenu de sa formation obligatoire.

\section{Remarques sur la production de levure aliment}

Dans des études précédentes, divers auteurs ont souligné que les souches appartenant à l'espèce de Kluyveromyces fragilis sont les mieux adaptées à la culture sur lactosérum $[1,2,4,7,10]$. Toutefois, les résultats qui précèdent montrent clairement que la culture continue d'une souche pure de Kluyveromyces fragilis peut s'accompagner d'une faible production d'éthanol et de la non-assimilation de l'acide lactique. En effet, en admettant un taux de dilution compatible avec la vitesse de croissance sur lactose de la souche 


\section{TABLEAU 1}

Temps de génération $(\mathrm{Tg})$, taux népérien de croissance $\left(\mu=\frac{\log _{2}}{\mathrm{Tg}}\right)$

$$
\text { et rendements } \mathrm{R}=\frac{\text { matière sèche produite }}{\text { substrat consommé }}
$$

d'une souche de Kluyveromyces fragilis en croissance sur milieu YNB avec différents substrats

\begin{tabular}{|c|c|c|c|}
\hline & $\mathrm{Tg}$ & $\mu$ & $\mathrm{R}$ \\
\hline Glucose & $2 \mathrm{~h} 10$ & 0,34 & 0,36 \\
\hline Lactose & $2 \mathrm{~h} 10$ & 0,32 & 0,38 \\
\hline Galactose & 2 h 10 & 0,32 & 0,35 \\
\hline Acide lactique & $2 \mathrm{~h} 40$ & 0,26 & 0,30 \\
\hline Ethanol & 7 h 20 & 0,09 & 0,54 \\
\hline
\end{tabular}

étudiée ici (par exemple 0,32), il y aurait bien consommation du lactose mais accumulation d'éthanol et non utilisation de l'acide lactique éventuellement présent. Cette difficulté est inérante à la biologie des levures à activité fermentaire et notamment de l'espèce Kluyveromyces fragilis. Ce problème ne peut donc être recherché uniquement dans l'amélioration de l'apport d'oxygène ou dans la géométrie du fermenteur.

Certes, il convient que le système d'aération permette un bon transfert d'oxygène pour éviter le développement d'un métabolisme fermentaire. De même, l'amélioration des transferts d'oxygène dans un fermenteur permettrait d'augmenter la concentration en substrats et de ce fait la biomasse à l'équilibre ; mais en aucun cas, une géométrie nouvelle de fermenteur ne pourra empêcher la formation d'éthanol : cette formation dépend exclusivement du métabolisme de la souche dans des conditions d'aération maximale où l'oxygène n'est pas limitant.

Il est connu que, dans le cas de diauxie, la consommation totale des substrats carbonés utilisables exige de diminuer le taux de dilution de manière très sensible,. Ainsi, dans le cas de Saccharomyces cerevisiae, la consommation totale du glucose ne peut se produire qu'à des taux de dilution très bas [3]. Par exemple Knopfel [3] a montré que pour une concentration en glucose de $10 \mathrm{~g} / \mathrm{l}$ à l'entrée du fermenteur, l'éthanol apparaît pour un taux de dilution égal ou 
supérieur à 0,30. Dans le cas de Candida tropicalis Knopfel [3] a trouvé des résultats analogues malgré l'absence d'effet glucose. En fait, dans le cas de Candida tropicalis comme dans celui de Kluyveromyces fragilis, l'effet Pasteur est toujours insuffisant pour empêcher toute fermentation quel que soit l'apport d'oxygène.

Dans le cas d'une souche de Kluyveromyces fragilis en culture pure, la consommation totale du substrat carboné avec un rendement de 0,5 , valeur observée par divers auteurs $[1,2,4,6]$, ne pourrait être atteinte en culture continue qu'avec un temps de renouvellement supérieur ou égal à $7 \mathrm{~h}$. Or les installations des Fromageries Bel permettent d'obtenir un rendement de 0,5 avec un temps de renouvellement moyen de 3 h $30 \mathrm{~min}$. Il est évident que ce résultat ne peut être atteint que par une maîtrise parfaite de la flore du fermenteur et une bonne connaissance des mécanismes de régulation de la levure.

\section{CONCLUSION}

Les conditions de production de levure aliment à partir de lactosérum doux dans un fermenteur industriel sont généralement [5] :

- aération 1,3 V/V/min (volume d'air insufflé dans le fermenteur par unité de volume et par minute) ;

- température $38^{\circ} \mathrm{C}$;

- $\mathrm{pH} 3,5$ à 4 ;

- concentration en substrat $30 \mathrm{~g} / 1$.

Ces valeurs permettent de travailler non stérilement.

Contrairement à ce que les études des paramètres de croissance laissent prévoir $[4,6]$, la culture exclusive de l'espèce Kluyveromyces fragilis ne permet pas d'obtenir les performances optimales en culture continue : ou bien le taux de dilution devient très faible (moins de 0,12 ) ou bien le rendement baisse car l'éthanol formé n'est pas utilisé.

L'utilisation d'une flore bien adaptée dans des conditions respectant les mécanismes de régulation de la levure ont cependant permis aux Fromageries Bel La Vache qui Rit d'obtenir un rendement de 0,52 avec un taux de dilution de 0,32 [5].

Les conditions expérimentales permettant de contourner les difficultés liées à la régulation des métabolismes des levures seront décrites ultérieurement.

\section{Rés u mé}

La production de levure aliment à partir de lactosérum fait appel le plus souvent à une culture pure de Kluyveromyces fragilis. 
L'étude comparative des potentialités de cette souche, avec les résultats obtenus à l'échelle industrielle, montre que ces derniers sont nettement supérieurs à ceux observés à l'échelle laboratoire. L'étude physiologique présentée permet de souligner l'importance sur le rendement d'un effet Pasteur insuffisant pour bloquer totalement la production d'alcool, même en présence d'une forte aération et bien que la souche ne présente pas d'effet glucose, vis-à-vis du métabolisme respiratoire. Il s'ensuit en culture pure une perte de rendement due à la production d'alcool.

\section{Su m m a ry}

\section{STUDY ON PHYSIOLOGICAL PROPERTIES OF KLUYVEROMYCES FRAGILIS: CONSEQUENCES ON THE PRODUCTION OF S.C.P. FROM WHEY}

The production of single cell proteins (S.C.P.) from whey, is performed with continuous culture of Kluyveromyces fragilis. This strain, does not show any crabtree effect, but the Pasteur effect is very strong; the fermentative activity is never absent even when strong aeration is present. So in all cases, alcohol is present in the medium. This observation can explain why the yield in an industrial production with an appropriate mixture culture is always higher than the yield in pure culture of Kluyveromyces fragilis.

Recu pour publication en décembre 1980.

\section{Bibliographie}

[1] Amundson (C. H.) (1967). - Increasing protein content of whey. Am. Dairy Rev., 29, 94-99.

[2] Chapman (L. P. J.) (1966). - Food yeast from whey. New Zealand J. of Dairy Technology, 1, 78-81.

[3] KNopfel (1974). - Zur Atmung und Gärung bei Hefen. Schweizer Brauerei Rundschau, 85, 1-14.

[4] Ladet (J.), Moulin (G.), Galzy (P.), Joux (J. L.) et Buju-Duval (F.) (1972), Comparaison des rendements de croissance sur lactose de quelques Kluyveromyces van der Walt. Le Lait, $\mathrm{n}^{\circ}$ 519-520.

[5] Marzolf (J. J.) (1977), - Point sur la situation actuelle de production de levures cultivées sur lactosérum. Colloque APRIA-DGRST. Les lactosérums, une richesse alimentaire, p. 73-82.

[6] Moulin (G.) et Galzy (P.) (1976). - Une possibilité d'utilisation du lactosérum : la production de levure. Ind. Alim. Agric., 11, 1337-1343.

[7] Moulin (G.) and Galzy (1978), - Remarks on the metabolism of Kluyveromyces lactis van der Walt. Mycopathologia, 66, 73-76.

[8] Slonimski (P.P.) (1955). - Adaptation respiratoire : développement du système hémoprotéique induit par l'oxygène. Proc. Third. Int. Congress Biochem. Brussels, Acad. Press Incorp., New York.

[9] Umbreit (W. W.), Burris (R. H.) and Stauffer (J. F.) (1964). - Manometric techniques. Fourth edition Burgess Publishing Company.

[10] Wasserman Aaron (E.) (1960). - The rapid conversion of whey to yeast. Dairy Engineering, 77, 374-379. 